\title{
Transmission Characteristics of Quarter Wave Plate Circular Polarizer Based on Frequency Selective Surface for High Frequency Applications
}

\author{
F.A. Mangi, S.Q. Xiao, Y.Q. Wang, D.A Jamro \\ School of Physical Electronics University of Electronic \\ Science \& Technology of China \\ Chengdu, China
}

\author{
I. Memon \\ College of computer science, Zhejiang University \\ Hangzhou, Zhejiang, China
}

\begin{abstract}
The broadband performance of quarter wave plate circular polarizer based on FSS is presented for high frequencies applications. Currently, the transmission loss and broadband of circular polarizer at desired frequencies is important issue for high frequencies application. Here, we are introducing some novel ideas to generate good circular polarization and significant techniques to improve broadband of circular polarization and low loss transmission by using single layer polarizer. The Simulation results show that the transmission loss $-2.4 \mathrm{~dB}$ of single layer polarizer has been achieved at $70.70 \mathrm{GHz}$ and pass band is extended from 69.29-71.78 GHz.
\end{abstract}

Keywords-frequency selective surface (FSS); quarter wave plate; dual layer polarizer; Fabry-Perot Interferometer; circular polarization

\section{INTRODUCTION}

The significance of detection and manipulation of the polarization state of electromagnetic waves have great interest in variety field of microwave and optical communication, wireless communication, radar and tracking applications [1-3]. Circular polarization has great importance because a circular polarization possesses the lower susceptibility to the reflection effects, atmospheric absorption and multipath. The circular polarization of waves is widely used in Satellite Communications Systems, Global Positioning System (GPS), and Radio Frequency Identification (RFID).

Circular polarizers based on meander line in microwave technology is introduced by Young [4] and presented by Munk [5]. Currently, Euler [6] has introduced the various types of dual layer circular polarizers in which the space between the surfaces of layers is adjusted by the Fabry Perot interferometer approach [7]. Quarter wave plate is another approach to transfer linearly polarized waves to circularly polarized waves. A quarter-wave plate converts electromagnetic waves from linearly to circularly polarised states or vice versa [8-14]. For a quarter wave plate, the transmitted phase difference of two orthogonal components of electric field is a quarter of a wavelength $\left(90^{\circ}\right)$. When an incident field is linearly polarized at $45^{\circ}$ and the quarter-wave plate converts the transmitted field to circular polarization, which has applications in satellite communication and rain clutter suppression [15].

For several years, Frequency Selective Surface has gained more attention in modern communication. Frequency selective surfaces (FSSs) are also used as polarizers, polarization transformer, filters, band-pass hybrid radomes for radar cross section (RCS) controlling [16-21] due to contributing overall stable performance for different incidence angles, polarization states and offering the simple fabrication. FSSs are known as spatial electromagnetic filters which show transmission over a required frequency band to an incident electromagnetic wave [22-23].

The pass band through polarizer is important issue in the field of wireless communication system; For instance, previous several published contributions [24], it has been observed that the poor broadband of circular polarizer was achieved at $75 \mathrm{GHz}$. Nevertheless, Fabry Perot Interferometer approach is introduced to fabricate FSS polarizer to improve the pass band [25].Another recent published contribution in which the transmission loss of the polarizer has been reduced $0.3 \mathrm{~dB}$ at $70 \mathrm{GHz}$ but band pass has not been improved with respect to axial ratio [26]. Currently, the FSSs circular polarizers have narrow bandwidth performance with poor efficiency of transmission; therefore research is needed to improve the bandwidth performance with low loss transmission of polarizer to achieve perfect circular polarization.

In this study, the novel approach has been introduced to design a single layer polarizer for high frequencies application that is quite different from previous several published contribution. We have improved the band pass which is extended form 69.29-71.78 GHz. The FSSs polarizer has several advantages over at present available circular polarizes, such as, broadband efficiency, perfect circular polarization, simple structure, high polarization efficiency, and easily fabrication.

\section{SINGLE LAYER POLARIZER DESIGN}

In this paper circular polarizer based on single layer FSS is designed high frequency applications. In unit cell, single dipole structure is designed at the angle $-45^{0}$ along the $x, y$ direction, respectively. The length of single strip is $l$ and wide $w$. whereas, perfect electric conductor (PEC) is assigned to the strip. The periods in $x$ - and $y$-direction are $P_{x}$ and $P_{y}$, respectively. 


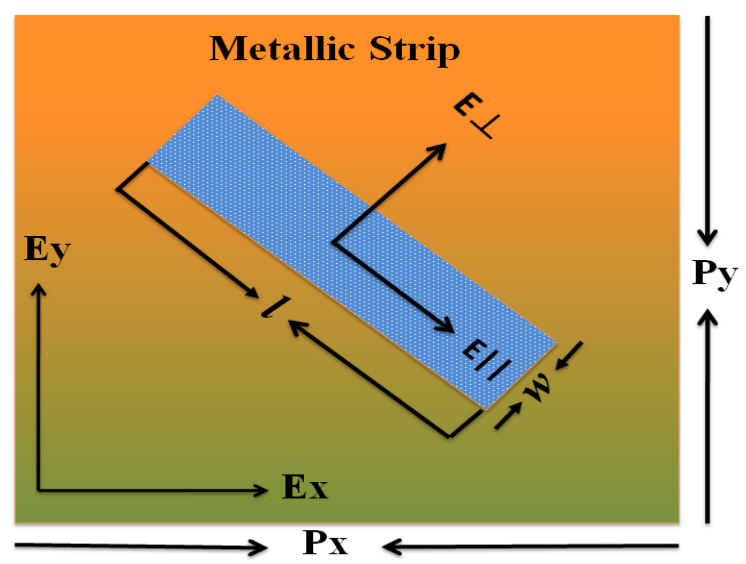

FIGURE I. THE DIMENSIONS OF SINGLE LAYER STRIP WITH ELECTRICAL FIELD ORIENTATION.

\section{Simulation SET UP}

The unit cell of single layer FSS polarizer is depicted in Fig. 2. The periodic boundaries are selected in $\mathrm{x}$ and $\mathrm{y}$ direction. Whereas, $\mathrm{x}$ polarized wave is used as the excitation source through floquent port one of this model to achieve circular polarization. The frequency domain solver HFSS-15.0 is used to simulate the single layer FSS with respect to periodic boundary conditions as shown in Fig.2. The structure parameters are selected as $l=3.6 \mathrm{~mm}, w=2.14 \mathrm{~mm}$ and $\mathrm{Px}=$ $8.4 \mathrm{~mm}$ and $\mathrm{Py}=8.4 \mathrm{~mm}$.

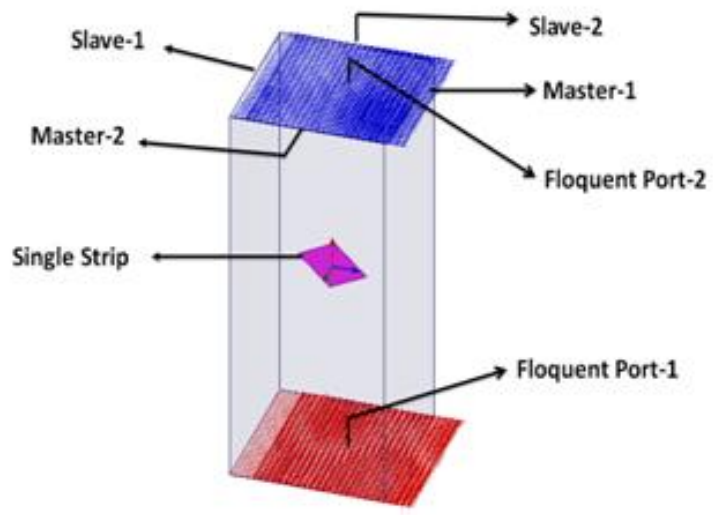

FIGURE II. SIMULATION MODEL OF SINGLE LAYER POLARIZER.

\section{A. Simulation Results \& Discussion}

When electromagnetic waves incident on the first layer the output E field from strip can be decomposed in to two orthogonal and linear electric field vector components (E\| and $\mathrm{E} \perp$ ) with equal magnitude. After EM waves passing through polarizer, the phase difference $90^{\circ}$ between two transmitted orthogonal components $(\mathrm{ET} \|$ and $\mathrm{ET} \perp$ ) can be produced with perfect circular polarization.

The probes of HFSS are used to calculate the output required parameters. After completing the simulation, the export based processing option is used to calculate the phase difference and the axial ratio of E components Ex and Ey while the transmission magnitudes and Phase shift of E field can directly be determined by the expression of Ex/Ey and $\Delta \phi$ $=\operatorname{Ex} \phi-\operatorname{Ey} \phi$.

The transmission loss is observed of the single layer polarizer where the radiated orthogonal components are same at the $70.70 \mathrm{GHz}$ but the transmission loss about $-2.4 \mathrm{~dB}$ is noticed as shown in Fig.3.

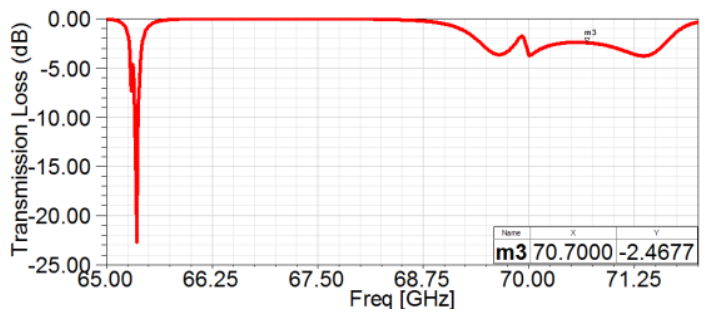

FIGURE III. TRANSMISSION MAGNITUDE OF EX AND EY OF EFIELD VERSUS FREQUENCY.

The phase difference between two orthogonal components is required $90^{\circ}$ to satisfy the perfect circular polarization. Here, the phase shift of two orthogonal components Ex and Ey is $90^{\circ} .20$ at the $70.70 \mathrm{GHz}$ to obtain the transmitted wave are perfect circular polarization that is equal to the transmission axial ratio between two orthogonal components as shown in Fig.4

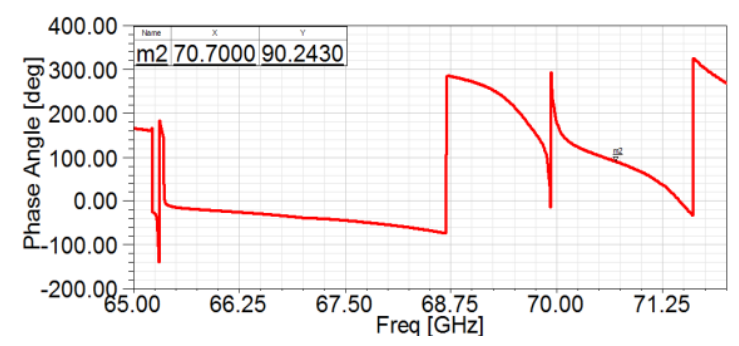

FIGURE IV. PHASE DIFFERENCE OF E-FIELD VERSUS FREQUENCY.

The magnitude of orthogonal components Ex and Ey remain same at the resonance frequency after transmission through FSS polarizer. The simulated axial ratio of circular polarizer is 1.2 that is equal to the phase difference 90.20 at 70.70 and pass band extends from $69.29-71.78 \mathrm{GHz}$ as shown in Fig: 5.

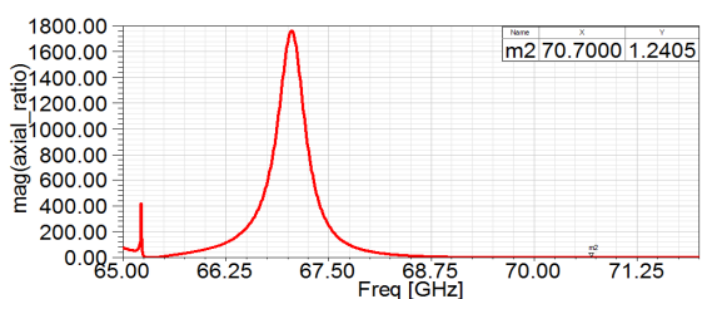

FIGURE V. SIMULATED AXIAL RATIO VERSUS FREQUENCY.

The most significant feature of this proposed designed model by using optimization techniques is to achieve the best results of FSS circular Polarizer. It is observed that the magnitudes of E component Ex and Ey are same at the frequency of $70.70 \mathrm{GHz}$ to obtain the perfect circular polarization. The pass band extends from $69.29-71.78 \mathrm{GHz}$. The phase difference of Ex and Ey is 900 and the magnitude 
values of Ex and Ey are same at $70.70 \mathrm{GHz}$ frequency, so approximately $100 \%$ circular polarization is achieved. Meanwhile, calculated values of circular polarization is deduced by the following equation (1) and verified by HFSS Software.

$$
\Delta \emptyset=\varnothing \mathrm{y}-\emptyset \mathrm{x}=\left\{\begin{array}{l}
+\left(\frac{1}{2}+2 \mathrm{n}\right) \pi, \mathrm{n}=0,1,2, \ldots \ldots \\
-\left(\frac{1}{2}+2 \mathrm{n}\right) \pi, \mathrm{n}=0,1,2, \ldots \ldots
\end{array}\right.
$$

\section{CONCLUSION}

The transmission characteristics of single layer polarizer has been investigated and achieved good circular polarization at $70.70 \mathrm{GHz}$. The best simulation results with good circular polarization on the pass band of $69.29-71.78 \mathrm{GHz}$ frequency have been obtained with low loss transmission $-2.4 \mathrm{~dB}$. The designed model is very simple and can be easily fabricated. Therefore, the same technique can be applied to fabricate single layer polarizers to enhance the bandwidth and minimize the transmission loss for millimetre, micrometre and terahertz frequencies according to desired applications. Nevertheless, it could possible by changing some parameters, such as by extending the dual, triple and multilayer, increasing or decreasing the length of each strips, adjusting the distance between layers and thickness of the layers.

\section{ACKNOWLEDGEMENT}

This work was supported in part by National Natural Science Foundation of China under Grants 61271028 and 61271027, in part by Fok Ying Tong Education Foundation under Grant 131107 and in part by Fundamental Research Funds for the Central Universities under Grant ZYGX2011J036.

\section{REFERENCES}

[1] J. H. Lee, Y. W. Song, J. G. Lee, J. Ha, K. H. H. wang, and D. S. Zang, "Optically bifacial thin-film wire-grid polarizers with nano-patterns of a graded metal-dielectric composite layer," Opt Exp. vol. 16, pp. 1686716876, October 2008.

[2] K. Iwami, M. Ishii, Y. Kuramochi, K. Ida, and N. Umeda, "Ultrasmall radial polarizer array based on patterned plasmonic nanoslits," Appl. Phys. Lett. vol. 101 , pp. 161119-1-4, 2012

[3] W. Li, D. M. Liu, and D. X. Huang, "Design of PM Polarizer Based on SiO2/ Si PLC Waveguide," Chinese Journal of Semiconductors. vol. 25, pp.1311-1314, 2004.

[4] L. Young, L. Robinson, and C. Hacking, "Meander-line polarizer,"IEEE Transactions on Antennas and Propagation, vol. 21, pp. 376-378, May 1973.

[5] B. A. Munk, Finite Antenna arrays and FSS. New York: Wiley, 2003.

[6] M. Euler, V. Fusco, R. Cahill, and R. Dickie, "Comparison of frequencyselective screen-based linear to circular split-ring polarisation convertors," IET Microwaves, Antennas and Propagation, vol. 4, pp. 1764 - 1772, November 2010.

[7] A. de C. Lima and E. Parker, "Fabry-perot approach to the design of double layer FSS," IEE Proceedings: Microwaves, Antennas and Propagation, vol. 143, pp. 157 - 162, April 1996.

[8] D. Lerner, "A wave polarization converter for circular polarization," IEEE Trans. Antennas Propag., vol. AP-13, no. 1, pp. 3-7, Jan. 1965.

[9] L. Young, L. Robinson, and C. Hacking, "Meander-line polarizer," IEEE Trans. Antennas Propag., vol. AP-21, no. 3, pp. 376-378, Mar. 1973.

[10] R.-S. Chu and K.-M. Lee, "Analytical method of a multilayered meander-line polarizer plate with normal and obliqueplane-wave
incidence,'IEEE Trans. Antennas Propag., vol. AP-35,

no.6, pp. 652-661, Jun. 1987.

[11] A. K. Bhattacharyya and T. J. Chwalek, "Analysis of multilayered meander line polarizer,'Int. J. Microw. Millimeter-Wave Comput.-Aided Eng., vol. 7, no. 6, pp. 442-454, 1997.

[12] M. Euler, V. Fusco, R. Cahill, and R. Dickie, "Comparison of frequencyselective screen-based linear to circular split-ring polarisation convertors," IET Microw., Antennas, Propag., vol. 4, no. 11, pp. 1764 1772, 2010.

[13] M. Euler, V. Fusco, R. Cahill, and R. Dickie, "325 GHz single layer submillimeter wave fss based split slot ring linear to circular polarization convertor," IEEE Trans. Antennas Propag., vol. 58, no. 7, pp. $2457-$ 2459, Jul. 2010.

[14] M. Joyal and J. Laurin, "Analysis and design of thin circular polarizers based on meander lines,"IEEE Trans. AntennasPropag., vol. 60, no. 6 , pp. 3007-3011, Jun. 2012

[15] Carl Pfeiffer and Anthony Grbic "Millimeter-Wave Transmitarrays for Wavefront and Polarization Control" IEEE Transactions on Microwave Theory and Techniques, vol. 61, no. 12, pp, 4407, december 2013,

[16] B. A. Munk, Frequency Selective Surfaces, Theory and Design, John Wiley \& Sons Inc, 2000

[17] G. I. Kiani, A. R. Weily, and K. P. Esselle, "A novel absorb/transmit FSS for secure indoor wireless networks with reduced multipath fading," IEEE Microwave and Wireless Components Letters, 16, pp. 378-380, 2006.

[18] G. I. Kiani, K. L. Ford, K. P. Esselle, A. R. Weily, and C. J. Panagamuwa, "Oblique incidence performance of a novel frequency selective surface absorber," IEEE Transactions on Antennas and Propagation, 55, pp. 2931-2934, 2007.

[19] A. Strikewerda et al.,"Comparison of birefringent metamaterials and meaderline structure as quarter-wave plates at terahertz frequencies," Optics Express, 17, pp. 136-149, 2009.

[20] K. Karkkainen, and M. Stuchly, "Frequency selective surface as a polarisation transformer," IEE Proceedings on Microwaves, Antennas and Propagation, 149, pp. 248-252, 2002.

[21] S. A. Winkler, W. Hong, M. Bozzi, and K. Wu, "A novel polarization rotating frequency selective surface based on substrate integrated waveguide technology," In Proceedings of the 39th European Microwave Conference, Rome, Italy, 2009.

[22] B. A. Munk, Frequency Selective Surfaces: Theory and Design.New York: Wiley, 2000.

[23] T. K. Wu, Frequency Selective Surface and Grid Array. New York: Wiley, 1995.

[24] G. I. Kiani and V. Dyadyuk, "Qurter-wave polariser based on frequency selective surfaces", Micow. conf. (EuMC), pp. 1361-1364, Sept 2010.

[25] A. C. De, C. Lima and E. A. Parker, "Fabry-Perot approach to the design of double layer FSS", IEE Proceedings on Microwaves, Antennas and Propagation, 143, 2, pp. 157-162, April, 1996.

[26] Ghaffer Kiani and Val Dyadyuk, "Low Loss FSS Polarizer for $70 \mathrm{GHz}$ Applications" IEEE Antennas and Propagation Society International Symposium (APSURSI), pp,1-2, 2012. 